\title{
Whole-Systems Assessment of the Value of Energy Storage in Low-Carbon Electricity Systems
}

\author{
Danny Pudjianto, Member, IEEE, Marko Aunedi, Student Member, IEEE, \\ Predrag Djapic, Member, IEEE, Goran Strbac, Member, IEEE,
}

\begin{abstract}
Energy storage represents one of the key enabling technologies to facilitate an efficient system integration of intermittent renewable generation and electrified transport and heating demand. This paper presents a novel whole-systems approach to valuing the contribution of grid-scale electricity storage. This approach simultaneously optimises investment into new generation, network and storage capacity, while minimising system operation cost, and also considering reserve and security requirements. Case studies on the system of Great Britain (GB) with high share of renewable generation demonstrate that energy storage can simultaneously bring benefits to several sectors, including generation, transmission and distribution, while supporting real-time system balancing. The analysis distinguishes between bulk and distributed storage applications, while also considering the competition against other technologies, such as flexible generation, interconnection and demand-side response.
\end{abstract}

Index Terms-Energy storage, Dispersed storage and generation, Power system planning, Power generation scheduling, Power system economics, Wind power generation.

\section{NOMENCLATURE}

\section{A. Constants}

$\alpha_{\mathrm{d}} \quad$ Ratio of flexible electricity demand to total demand

$\alpha_{d}^{r s p} \quad$ Proportion of flexible loads that can be interrupted to provide frequency response

$\alpha_{d}^{\text {res }} \quad$ Proportion of flexible loads that can be interrupted to provide operating reserves

$\alpha_{s}^{r s p} \quad$ Proportion of storage charging that can be interrupted to provide frequency response

$(\alpha, \beta)_{L, n} \quad$ Linear coefficient and constant term for the $n$-th piecewise linear approximation of LOLP function Demand-Side Response (DSR) efficiency [\%]

$s \quad$ Storage efficiency [\%]

$\bar{\mu} \quad$ Number of existing generating units

$\pi_{\overline{d n}} \quad$ Distribution network reinforcement cost per unit

$\pi_{\hat{f}} \quad$ Transmission network reinforcement cost per unit

$\pi_{g} \quad$ Generation operating cost per unit

$\pi_{\hat{\mu}} \quad$ Generation investment cost per unit

$\pi_{n l} \quad$ Generation no-load-cost

$\pi_{\hat{s}} \quad$ Storage investment cost per unit

$\pi_{s t} \quad$ Generation start-up cost

D. Pudjianto, M. Aunedi, P. Djapic and G. Strbac are with the Departmen of Electrical Engineering, Imperial College London, United Kingdom (e-mail: \{g.strbac, m.aunedi, d.pudjianto, p.djapic\}@imperial.ac.uk).

\section{B. Variables}

$\theta \quad$ Voltage angle

$\mu \quad$ Number of units in operation

$\hat{\mu} \quad$ Number of additional generating units installed

$d_{+} \quad$ Increased electricity load due to DSR [MW]

$d_{-} \quad$ Reduction in electricity load due to DSR [MW]

$\widehat{d n} \quad$ Additional distribution network capacity [MW]

$d s \quad$ Number of generating units being de-synchronised

es $\quad$ Energy content of storage [MWh]

$\hat{f} \quad$ Additional transmission network capacity [MW]

$g \quad$ Electricity production [MW]

res Spinning reserve provided by generators [MW]

rsp Frequency response provided by generators [MW]

$s_{+} \quad$ Electricity generated by storage [MW]

$s_{-} \quad$ Electricity consumed by storage [MW]

$\hat{s} \quad$ Additional storage capacity [MW]

st Number of generating units being synchronised

$C M \quad$ Capacity margin [MW]

LOLP Estimated Loss of Load Probability (LOLP)

C. Functions

$C_{d n}(\cdot) \quad$ Piecewise linear distribution network reinforcement cost function

$C_{g}(\cdot) \quad$ Generation operating cost function

$F(\cdot) \quad$ Power flows function 


$\begin{array}{ll}D . \text { Sets } & \\ D & \text { Set of electricity loads } \\ D N & \begin{array}{l}\text { Set of distribution networks; } D N_{i} \text { is a set of compo- } \\ \text { nents belonging to distribution network } i\end{array} \\ & \text { Set of operating snapshots on the } x \text {-th day } \\ D_{x} & \text { Set of transmission/interconnection corridors } \\ F & \text { Set of generators } \\ G & \text { Set of storage devices } \\ S & \text { Set of operating snapshots }\end{array}$

\section{INTRODUCTION}

$\mathrm{G}^{\circ}$ OVERNMENTS around the world are committed to substantially reducing energy system driven greenhouse gas emissions in the coming decades. As part of this effort electricity systems are expected to integrate significant amounts of intermittent renewable generation in combination with less flexible nuclear and Carbon Capture and Storage plant, while large segments of the transport and heat sectors are expected to be electrified.

Integration of intermittent generation characterised by a low capacity value, accompanied with potentially substantial increases in peak demand driven by transport and heating electrification, may lead to very significant degradation in the utilisation of generation infrastructure and electricity network assets. Furthermore, operating reserve requirements and the need for flexibility at high penetration of intermittent renewable generation increase significantly above those in the conventional systems. This in turn will decrease the ability of the system to absorb intermittent generation, if these services are provided through traditional generation based resources. This becomes a major problem during off-peak hours, particularly if it coincides with high renewable output, which may lead to highly undesirable curtailment of renewable output. As a result, system integration costs are expected to increase considerably.

Energy storage technologies have the potential to support future system integration of renewable energy. However, the potential value that storage brings to the system, and therefore its cost targets, are currently not fully understood, especially in terms of the ability of storage to simultaneously deliver benefits in multiple segments of the electricity system, including distribution networks, transmission networks and interconnection, real time demand-supply balancing and supply adequacy.

Previous research on the value of storage for integrating intermittent renewable generators mostly focused on the capability of storage to perform arbitrage [1] or provide reserve [2] in systems characterised by high penetration of intermittent generation. In that respect, a framework for assessing the benefits and market potential of storage for utility-related applications is outlined in [3]. Specific uses of energy storage systems to manage the output variability of wind and solar generation are addressed in [4] and [5]. Stochastic approaches to valuation of storage for arbitrage and reserve, such as the one provided in [6], are particularly suitable for studying the value of storage in systems with large shares of wind generation. Previous work investigated a number of different storage technologies and their potential applications, such as: stochastic optimisation of pumped-storage units [7] or compressed air energy storage [8] to support market participation of wind generation; grid-scale application of batteries [9]; or sizing and control of flow batteries [10].

Reliability benefits of energy storage in a system with high wind penetration including the improvement of wind capacity credit are quantified in [11] and [12], while the problem of optimal sizing of storage systems is addressed in [13] and [14]. The potential of storage to contribute simultaneously to both energy markets and frequency regulation is evaluated in [15] and [16].

Various control approaches for distributed storage have been proposed in [17] and [18], based on the objectives of reducing losses, improving the voltage profile, and enabling post-fault load restoration. The net present value (NPV) of storage installations in distribution substations is quantified in [19] as a function of unit costs of storage power and energy. References [20] and [21] analyse the impact of storage on improving the economic performance, reliability and the integration of renewable sources in a microgrid-based environment.

This paper presents a novel whole-electricity systems approach to valuing the contribution of grid-scale electricity storage. This model simultaneously optimises investments into new generation, distributed and bulk storage technologies, interconnection, transmission and distribution assets while minimising short-term operation cost of system through hourly system operation representation, while considering reserve and frequency regulation requirements. System adequacy and security requirements together with emission constraints are also considered within the same framework. The model further includes a detailed representation of electricity demand, and considers the capability of demand response technologies, using the inputs supplied by detailed bottom-up demand models.

This approach reveals trade-offs between multiple services that energy storage is able to provide, which result in generally higher aggregate values for storage than in previous approaches that considered such services in isolation.

The advantages of this approach are illustrated by modelling and analysing the value of grid-scale storage in the future GB electricity system. The paper aims at addressing some of the key questions in relation to the future role of electricity storage: What are the cost targets and scale of deployment? What are the benefits of storage across different time scales and different sectors of the system (from real time operation to investment time scale, considering generation, transmission and distribution sectors)? What type of storage delivers the highest value and where should it be placed on the network?

\section{WhOLE-SySTEMS APPROACH TO POWER SYSTEM ANALYSIS}

When considering system benefits of storage technologies it is important to consider two key aspects: (i) Different time horizons: from long-term investment-related time horizon to real-time balancing on a second-by-second scale; this is important as storage technologies can contribute to both savings in generation and network investment but also to increase the efficiency of system operation; and (ii) Different assets in the energy system: generation assets (from large-scale to distributed small-scale), transmission network (national and interconnections), and local distribution network operating at various 
voltage levels. This is important as storage technologies may be placed at different locations in the system, from bulk technologies connected to the national transmission network, such as large-scale storage to highly distributed technologies connected to local low-voltage distribution networks.

In this context, the novel whole-systems approach proposed in this paper simultaneously optimises investments into new generation, storage, interconnection, transmission and distribution assets while considering short-term operation of the entire system on an hourly basis. System adequacy and security requirements together with emission constraints are considered within the same framework. The model further includes a detailed representation of electricity demand, and considers the capability of demand response technologies, using the inputs supplied by our detailed bottom-up demand models.

It should be noted that energy storage in the context of this paper refers to dedicated storage installations for grid applications (either bulk or distributed), but does not include demandside technologies with storage capabilities such as electric vehicles (EVs), as these technologies are not deployed solely for the purpose of storing electricity. These technologies are however considered in this analysis as part of DSR.

Due to a great variety in parameters of different storage technologies, a technology-agnostic approach has been adopted in this paper where no particular choice is made with respect to storage technology. The objective is rather to assess the system value of storage represented through generic parameters (power rating, duration, efficiency, installation cost etc.), while varying the value of these parameters in a rather broad range in order to cover a wide variety of storage technologies potentially available in the future.

The problem is formulated as a mixed integer linear programming problem with the time horizon of 1 year and hourly time resolution. The objective function (1) is to minimise the overall system cost, which consists of annuitised investment cost associated with various generation, network and storage assets and the annual operating cost:

$$
\begin{aligned}
\text { Minimise } \varphi=\sum_{i=1}^{G} & \pi_{\widehat{\mu}_{i}} \cdot \hat{\mu}_{i}+\sum_{i=1}^{S} \pi_{\hat{s}_{i}} \cdot \hat{s}_{i}+\sum_{i=1}^{F} \pi_{\hat{f}_{i}}, \hat{f}_{i} \\
& +\sum_{i=1}^{D N} C_{d n}\left(\pi_{\widehat{d n}_{i}}, \widehat{d n}_{i}\right) \\
& +\sum_{t=1}^{T} \sum_{i=1}^{G} C_{g_{i}}^{t}\left(\pi_{g_{i}}, g_{i}^{t}, \pi_{n l_{i}}, \pi_{s t_{i}}, \mu_{i}^{t}\right)
\end{aligned}
$$

The investment cost includes (annuitised) capital cost of new generating and storage units, capital cost of additional transmission and interconnection capacity, and the reinforcement cost of distribution networks. Various types of investment costs are annuitised using the appropriate WeightedAverage Cost of Capital (WACC) and the estimated economic life of the asset. Both of these parameters are provided as inputs to the model for each technology.

System operating cost is the total annual generation cost that consists of: (i) variable cost which is a function of electricity output, (ii) no-load cost which is a function of a number of synchronised units, and (iii) start-up cost. These operating cost categories have been modelled using the approach presented in [22]. The effect of carbon prices has been calculated into the operating cost.

There are a number of equality and inequality constraints that are taken into account by the model while minimising the overall cost. All constraints are applied for each time interval within the optimisation time horizon $(\forall t \in T)$. These include:

Power balance constraints (2), which ensure that supply and demand, taking into account storage and DSR, are balanced at all times.

$$
\sum_{i=1}^{G} g_{i}^{t}+\sum_{i=1}^{S}\left(s_{+i}^{t}-s_{-i}^{t}\right)-\sum_{i=1}^{D}\left(\mathrm{~d}_{i}^{t}+d_{+i}^{t}-d_{-i}^{t}\right)=0
$$

Generator operating constraints include: (i) Minimum Stable Generation (MSG) and maximum output constraints (3); (ii) ramp-up (4) and ramp-down (5) constraints; (iii) minimum up (6) and down time (7) constraints; (iv) available frequency response and reserve constraints (8); maximum response constraints for each generation technology (9); annual load factor constraints (10); and the maximum number of synchronised units (11). Constraints (3)-(11) are applied to all generators $(\forall i \in G)$.

$$
\begin{gathered}
\mu_{i}^{t} \cdot \underline{g}_{i} \leq g_{i}^{t} \leq \mu_{i}^{t} \cdot \bar{g}_{i} \\
g_{i}^{t}-g_{i}^{t-1} \leq \mu_{i}^{t} \cdot \mathrm{r}_{u p_{i}} \\
g_{i}^{t-1}-g_{i}^{t} \leq \mu_{i}^{t-1} \cdot \mathrm{r}_{d n_{i}} \\
\sum_{k=t-\underline{U p}_{i}}^{t-1} s t_{i}^{k} \leq \mu_{i}^{t} \\
\mu_{i}^{t} \leq \bar{\mu}_{i}+\hat{\mu}_{i}-\sum_{k=t-\underline{D n}_{i}}^{t-1} d s_{i}^{k} \\
\mu_{i}^{t} \cdot \underline{g}_{i} \leq g_{i}^{t}+r s p_{i}^{t}+r e s_{i}^{t} \leq \mu_{i}^{t} \cdot \bar{g}_{i} \\
r s p_{i}^{t} \leq \mu_{i}^{t} \cdot \overline{\operatorname{rsp}}_{i} \\
\sum_{t=1}^{T} g_{i}^{t} \leq \mathrm{LF}_{i} \cdot \tau \cdot\left(\bar{\mu}_{i}+\hat{\mu}_{i}\right) \cdot \overline{\mathrm{g}}_{i} \\
\mu_{i}^{t} \leq \bar{\mu}_{i}+\hat{\mu}_{i}
\end{gathered}
$$

The model optimises both the quantity and the location of new generation capacity for various generation technologies. If required, a set of constraints can be put in place to limit the investment in particular generation technologies at given locations. Annual load factor constraints (10) can be used to limit the utilisation level of thermal generating units, e.g. to account for the effect of planned annual maintenance on plant utilisation.

Storage operating constraints include: maximum power rating constraints for storage charging (12) and discharging cycles (13); constraints associated with the amount of energy that can be stored (14); and the storage energy balance (15). As implied in (12)-(14), the model considers new investment in energy storage by optimising its location and capacity to minimise the overall cost (1). Constraints (12)-(15) are applied to all storage units $(\forall i \in S)$.

$$
\begin{gathered}
s_{+i}^{t} \leq \overline{\mathrm{s}}_{i}+\hat{s}_{i} \\
s_{-i}^{t} \leq \overline{\mathrm{s}}_{i}+\hat{s}_{i} \\
e s_{i}^{t} \leq\left(\overline{\mathrm{s}}_{i}+\hat{s}_{i}\right) \cdot \mathrm{sc}_{i} \\
e s_{i}^{t}=e s_{i}^{t-1}-s_{+i}^{t}+{ }_{s_{i}} \cdot s_{-i}^{t}
\end{gathered}
$$

Demand-side response constraints include constraints for various specific types of loads. Different demand categories are associated with different levels of flexibility. Flexibility 
parameters associated with various forms of DSR are obtained using detailed bottom-up modelling of different types of flexible demand, as described in [24] and [25] for EVs and heat pumps (HPs). A set of generic DSR constraints is presented below. These include the demand reduction constraints (16) and the energy balance for demand shifting (17) potentially considering losses driven by temporal shifting of demand (as shifting demand may increase the overall energy requirements), quantified through the efficiency ${ }_{d}$. Constraints (16)(17) are applied to all electricity loads $(\forall i \in D)$.

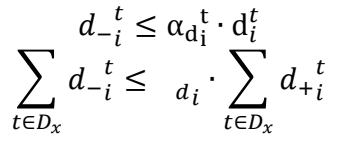

Operating reserve constraints include various forms of fast and slow reserve constraints. The amount of operating reserve requirement is calculated as a function of uncertainty in generation and demand across various time horizons. The model distinguishes between two key types of balancing services: (i) frequency regulation (response), which is delivered in the timeframe of a few seconds to 30 minutes; and (ii) reserve, typically split between spinning and standing reserve, with delivery occurring within the timeframe of half an hour to several hours after the request. The need for these services is directly driven by wind output forecasting errors and this will significantly affect the ability of the system to absorb wind energy. Calculation of reserve and response requirements for a given level of intermittent renewable generation is carried out exogenously and provided as input into the model.

The frequency response and reserve constraints are formulated in (18) and (19), respectively, stating that the contribution of all generators to response $(r s p)$ and reserve $(r e s)$, combined with the contributions from storage and DSR, needs to satisfy the system-level requirements for the two services.

$$
\begin{gathered}
\sum_{i=1}^{G} r s p_{i}^{t}+\sum_{i=1}^{S}\left(\alpha_{s, i}^{r s p} \cdot s_{-i}^{t}\right)+\sum_{i=1}^{D}\left\{\alpha_{d, i}^{r s p} \cdot\left(\mathrm{d}_{i}^{t}+d_{+i}^{t}-d_{-i}^{t}\right)\right\} \\
\geq \underline{\operatorname{srp}}^{t} \\
\sum_{i=1}^{G} r e s_{i}^{t}+\sum_{i=1}^{S}\left(\bar{s}_{i}+\hat{s}_{i}-s_{-i}^{t}\right)+\sum_{i=1}^{D}\left\{\alpha _ { d , i } ^ { r e s } \cdot \left(\mathrm{~d}_{i}^{t}+d_{+i}^{t}\right.\right. \\
\left.\left.-d_{-i}^{t}\right)\right\} \geq \underline{s r s}^{t}
\end{gathered}
$$

The amount of spinning and standing reserve and response is optimized ex-ante to minimise the expected cost of providing these services, and we use our advanced stochastic generation scheduling models to calibrate the amount of reserve and response scheduled in the model [6],[23]. These stochastic models find the cost-optimal levels of reserve and response by performing a probabilistic simulation of the actual utilisation of these services.

Power flow constraints (20) limit the energy flowing through the transmission system respecting the total installed capacity as the upper bound. The model optimises the location and capacity of new transmission investment to minimise the objective function. Power flows are calculated as a function of net power injection, network topology and parameters.

$$
-\left(\overline{\mathrm{f}}_{i}+\hat{f}_{i}\right) \leq F(G, S, D, \theta)_{i}^{t} \leq \overline{\mathrm{f}}_{i}+\hat{f}_{i} \quad \forall i \in F
$$

Due to the simplified representation of transmission network used in the studies, a standard linear power flow model is adopted. Power flow is a function of power injections by generation, load and storage, as well as of network topology and its parameters, as described in [26].

Expanding transmission and interconnection capacity is generally found to be vital for facilitating efficient integration of large intermittent renewable resources, given their location. Interconnectors provide access to renewable energy and improve the diversity of demand and renewable output on both side of the interconnector, thus reducing the short-term reserve requirement. Interconnection also allows for sharing of reserves, which reduces the long-term capacity requirements.

The model can both reinforce existing transmission links and add new capacity between previously unconnected regions (where allowed by the user). The model will reinforce both existing and new corridors if economically justified. Possible additions of new transmission lines between buses that are not initially connected would need to be specified by the user.

Distribution network peak load constraints (21): The whole-system assessment model requires the distribution network reinforcement cost to be expressed as a function of peak load in a given distribution system (driven by electrification of transport and heat sectors). This is informed by detailed modelling of representative networks. Different types of distribution networks based on the statistical models of urban, semiurban, semi-rural and rural networks [27],[28] are analysed, as well as different levels of penetration of EVs and HPs and their operating regimes. This approach allows for the quantification of respective reinforcement costs. As an example, the cost function for a generic urban network is presented in Fig. 1. If the model decides to install distributed storage, its operation will likely change the aggregate demand profile and consequently the necessary reinforcement cost.

As the network reinforcement cost function is likely to be non-linear and lumpy, a piecewise linear approximation of the cost function is proposed for the whole-system model formulation.

$$
\begin{array}{r}
\sum_{j \in D N_{i}} \mathrm{~d}_{j}^{t}+\sum_{j \in D N_{i}} d_{+}^{t}-\sum_{j \in D N_{i}} d_{-j}^{t}-\sum_{j \in D N_{i}} g_{j}^{t}-\sum_{j \in D N_{i}} s_{+}^{t} \\
+\sum_{j \in D N_{i}} s_{-j}^{t}-\overline{d n}_{i} \leq \widehat{d n}_{i} \quad \forall i \in D N
\end{array}
$$

Security constraints ensure that there is sufficient generating capacity in the system to supply the demand with a given level of security and estimates the Loss of Load Expectation (LOLE). Fig. 2 illustrates the use of piecewise linear functions to approximate the LOLP function. The LOLP curve, being a function of the capacity margin (CM), has to be built a priori for a given system using a standard reliability approach. The $\mathrm{CM}$ is defined as the ratio of surplus generating capacity (including storage when it produces electricity) and the peak demand. Sharing of capacity between interconnected regions can also be taken into account to increase the CM. 

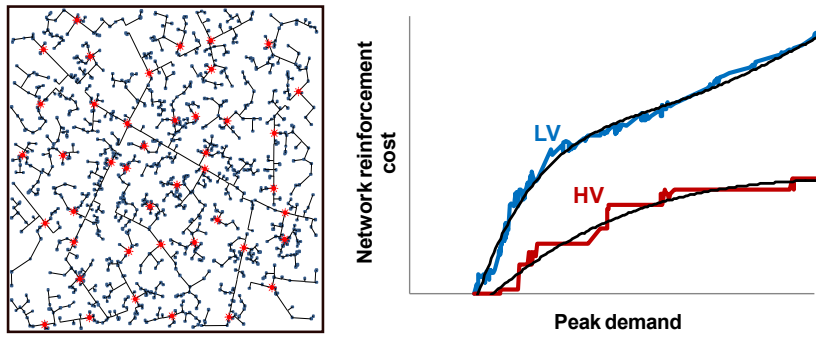

Fig. 1. A generic urban network with its low voltage (LV) and high voltage (HV) network reinforcement cost function for various peak demand conditions

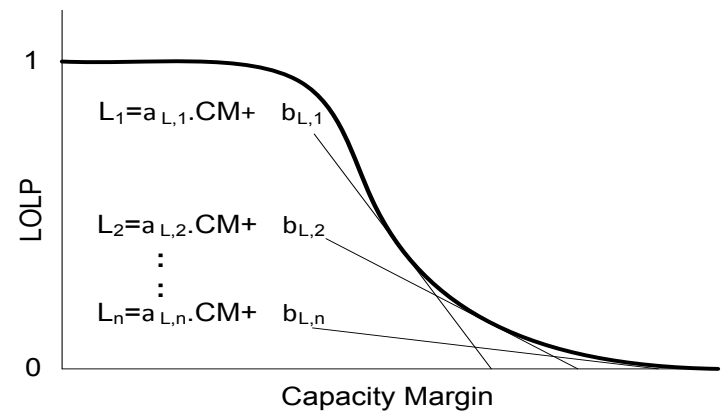

Fig. 2. Piecewise linear approximation of LOLP function

Constraints (22) are used to approximate the LOLP, and the sum of LOLP across the year should meet the reliability criterion as defined by $\overline{L O L E}(23)$.

$$
\begin{gathered}
\operatorname{LOLP}_{i}^{t} \geq \alpha_{L, 1} C M(\cdot)+\beta_{L, 1} \\
\operatorname{LOLP}_{i}^{t} \geq \alpha_{L, n} C M(\cdot)+\beta_{L, n} \\
\sum_{t=1}^{T} \operatorname{LOLP} P_{i}^{t} \leq \overline{\operatorname{LOLE}}_{i}
\end{gathered}
$$

The mixed integer linear problem defined in this section has been implemented in the FICO Xpress optimisation tool [29].

\section{CASE STUDY: LOW-CARBON UK SYSTEM}

\section{A. System Description}

We apply the proposed whole-system modelling approach on the UK system in 2030, a scenario developed by the UK government. The total contribution of renewable generation is $54.7 \%$ of the total annual energy demand, with the contribution of intermittent wind generation of $52.5 \%$, which corresponds to $68.7 \mathrm{GW}$ of installed wind capacity.

On the demand side, the 2030 renewable scenario is characterised by a fast electrification of transport and heating sectors, accompanied by highly ambitious measures in the area of improved building insulation and energy efficient consumer behaviour. In the heating sector about $50 \%$ of heat demand in the residential and commercial sectors is met by electricity, while $63 \%$ of road transport is expected to be based on EVs.

Winter peak demand is estimated at $104 \mathrm{GW}$ and minimum summer demand is $26 \mathrm{GW}$, with total annual electricity demand of $433.5 \mathrm{TWh}$. Given that the GB transmission network is characterised by significant North-South power flows, for the purpose of this study the GB system was represented using 5 key regions and their boundaries: 1) Scotland, 2) North England and Wales (EW-N), 3) Middle England and Wales (EW$\mathrm{M})$, 4) South England and Wales (EW-S), and 5) London (embedded within the South England and Wales region). The topology of this system is depicted in Fig. 3. The two neighbouring systems, Ireland (IE) and Continental Europe are represented as separate areas, with an option to link Ireland directly to mainland Europe. Network lengths in Fig. 3 reflect the equivalent distances which take into account the additional local network investment that interconnection may require. Network capacities indicated in the figure refer to the capacities expected to be in place by $2020{ }^{1}$

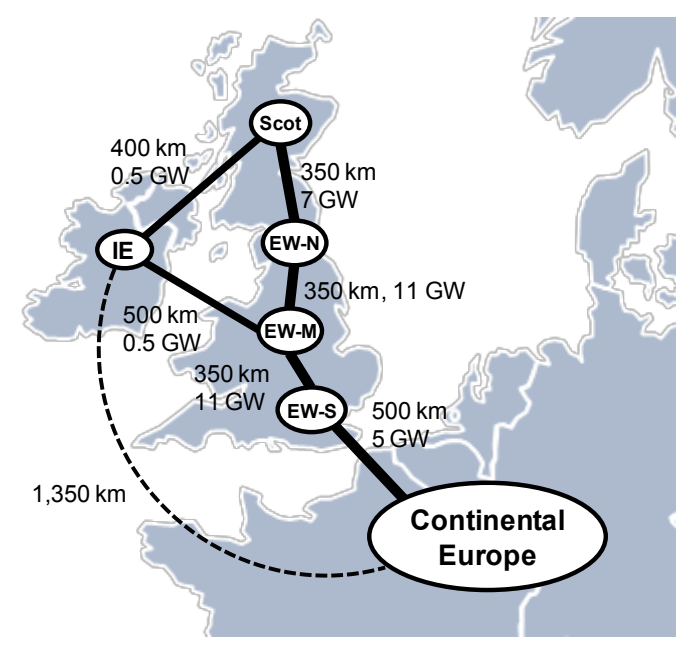

Fig. 3. Topology of the interconnected GB system used in the study

Both economic and reliability considerations are involved in transmission network and interconnection design [30]. In each of the regions distribution networks are represented by a mix of 10 statistically representative distribution networks based on the approach developed in [31].

The model described in Section III determines the optimal volume of storage that should be deployed in each of the GB regions. In the case of distributed storage the model further decides how much storage capacity to install in each of the representative distribution networks associated with different areas. The user of model can also impose constraints regarding the amount of new storage that can be added in each of the regions and/or distribution networks, in order to reflect various external constraints, such as restrictions associated with land use.

As documented in [32], there is considerable uncertainty regarding the future costs of electricity storage. Annuitized storage installation costs in this paper have therefore been assumed within relatively broad ranges: $£ 50-400 / \mathrm{kW}$.year for bulk and $£ 100-500 / \mathrm{kW}$.year for distributed. A slightly higher range of installation costs associated with distributed storage reflect the effect of economies of scale, which is likely to make a unit of distributed storage capacity more expensive than bulk. Given the annual time horizon of the assessment model, which balances system operation with investment cost in networks, generation and storage the investment cost are represetn4ed through annuitized values rather than total capital cost.

Key assumptions on annuitized generation and network ca-

\footnotetext{
${ }^{1}$ No direct link was assumed to be in place between Ireland and continental Europe in 2020, but the model was allowed to build new capacity between the two systems if economically justified.
} 
pacity cost, as well as fuel and carbon cost based on UK national projections are provided in Table I. A number of other generator parameters was also used, in particular the dynamic parameters such as minimum up or down times, or no-load and start-up costs. Assumptions on the costs of distribution network assets have been taken from [31], and are omitted here for brevity.

TABLE I

ASSUMPTIONS ON INVESTMENT AND FUEL COSTS

\begin{tabular}{|c|c|c|c|}
\hline Technology & $\begin{array}{c}\text { Annuitised cost } \\
(£ / k W \text {.year })\end{array}$ & Commodity & Cost \\
\hline Nuclear & 352.9 & Coal & $\$ 110 / \mathrm{t}$ \\
\hline CCGT & 56.6 & Gas & $£ 0.70 /$ therm \\
\hline Coal & 133.9 & Oil & $\$ 130 /$ barrel \\
\hline CCGT CCS & 197.2 & Carbon permit & $£ 74.2 / \mathrm{t}$ \\
\hline Coal CCS & 431.6 & & \\
\hline OCGT & 47.5 & & \\
\hline HVDC transmission & £96.0/MW.km.year & & \\
\hline
\end{tabular}

\section{B. Benefit of Storage}

Fig. 4 and Fig. 5 present the net system benefits of bulk energy storage and distributed energy storage respectively, for storage durations of 6 and 24 hours. In each chart the composition of annual system benefits is given in £bn per year, for a range of assumed energy storage annuitised capital costs (top horizontal axis) also corresponding to different optimal volumes of storage proposed by our model (bottom horizontal axis). Components of system savings depicted include: operation cost (OPEX), generation investment (G CAPEX), transmission investment (T CAPEX), interconnection investment (IC CAPEX) and distribution investment (D CAPEX). Optimal level of investment into energy storage (S CAPEX) is plotted as negative benefit, resulting in a net system benefit that is also depicted in the figure ("Total"). The same notation is used in the remaining figures in the paper.

Net benefits from deploying storage, after discounting its investment cost from gross system benefits, reach up to about $£ 1.8 \mathrm{bn} /$ year for bulk storage available at the annuitised investment cost $£ 50 / \mathrm{kW}$.year (Fig. 4) and above $£ 2 \mathrm{bn} /$ year for distributed storage available at the annuitised investment cost $£ 100 / \mathrm{kW}$.year (Fig. 5). We note that the optimal storage volume increases as the storage investment cost drops, as expected.

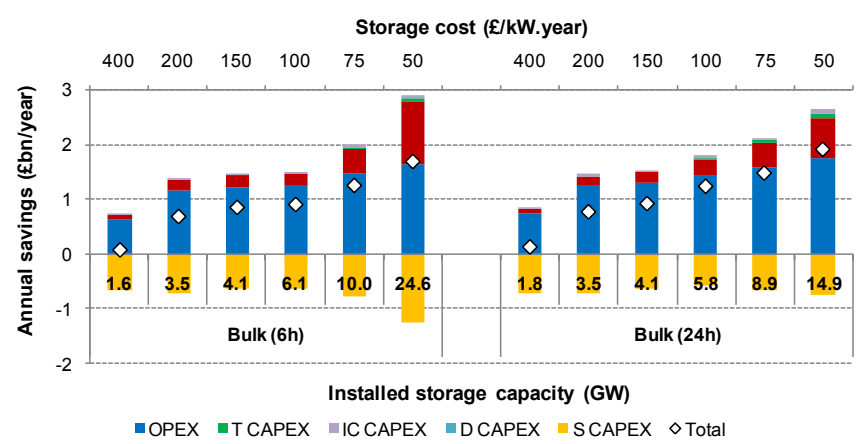

Fig. 4. Annual net benefit of bulk storage in 2030 for 6-hr and 24-hr duration

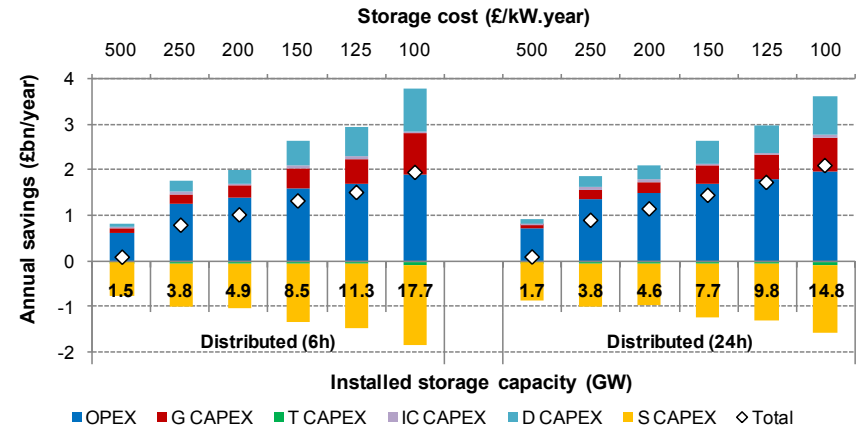

Fig. 5. Annual net benefit of distributed storage in $2030 \mathrm{for} 6-\mathrm{hr}$ and $24-\mathrm{hr}$ duration

We also notice that storage duration is a factor influencing the volume of storage deployed. For the cost of storage below the level of $£ 150 / \mathrm{kW}$.year for bulk i.e. $£ 200 / \mathrm{kW}$.year for distributed, higher durations suggest a lower optimal volume of storage deployed when available at the same cost (for higher storage cost there is no significant difference). This is a consequence of a larger energy that can be stored in a 24-hour storage when compared with e.g. 6-hour storage having the same installed capacity in GW. We observe that when 6-hr bulk storage is available at $£ 50 / \mathrm{kW}$.year, the optimal amount installed is $24.6 \mathrm{GW}$, while for distributed storage available at $£ 100 / \mathrm{kW}$.year, the optimal amount is $17.7 \mathrm{GW}$. In case that 24-hour storage is available at these cost levels, the total amount installed is reduced to $14.9 \mathrm{GW}$ for bulk and $14.8 \mathrm{GW}$ for distributed.

Also, for the same cost a lot more distributed storage capacity is deployed, when compared with bulk storage. Furthermore, even when distributed storage is more expensive than bulk (such as e.g. for the cost of bulk of $£ 150 / \mathrm{kW}$.year and the cost of distributed of $£ 200 / \mathrm{kW}$.year), the capacity of distributed storage added to the system can be higher.

\section{Value per Unit of Installed Storage Capacity}

We further present in Fig. 6 and Fig. 7 the average value of bulk and distributed energy storage respectively for the same cases as before, obtained by dividing the gross system benefits of storage for a particular cost case (not considering storage investment cost) with the optimal volume of storage deployed in that same case. This provides interesting insights into how a unit of storage $(1 \mathrm{~kW})$ performs in terms of providing benefits to the system.

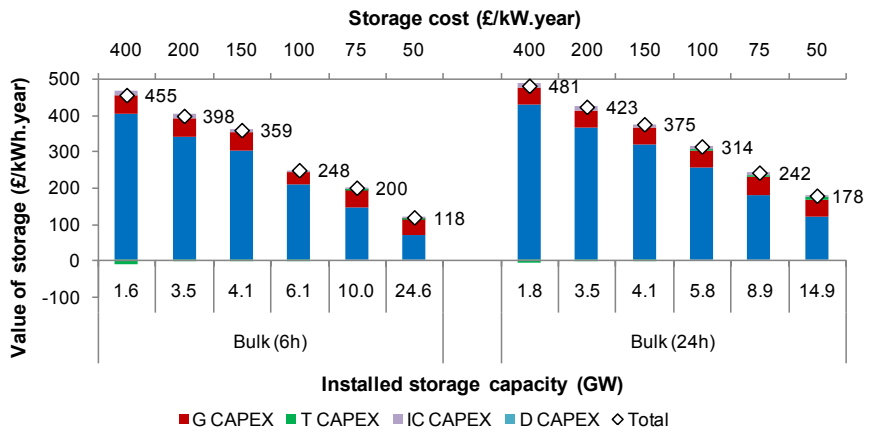

Fig. 6. Value of bulk storage (for 6-hr and 24-hr durations) 


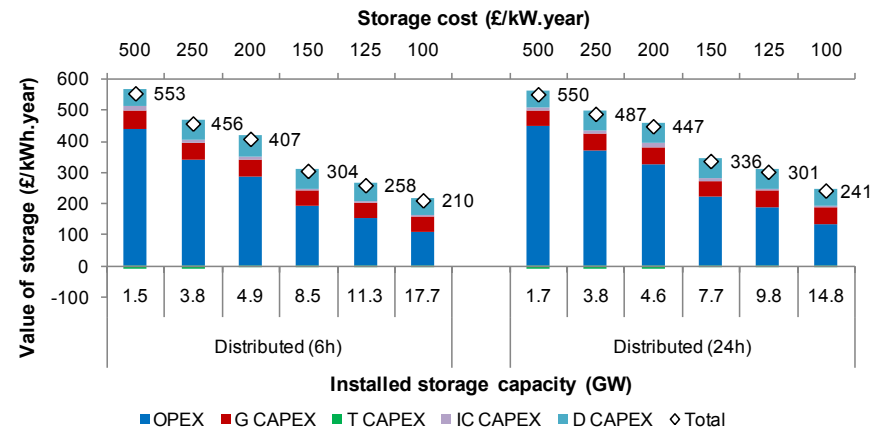

Fig. 7. Value of distributed storage (for 6-hr and 24-hr durations)

Savings in operating costs mostly result from reduced curtailment of wind generation and are a dominant component of the value of storage, although they diminish with higher storage penetration, as it becomes progressively more challenging to reduce operating costs. We note that across the range of annuitised investment costs for bulk and distributed energy storage there is a relatively stable generation investment cost saving of about $£ 50 / \mathrm{kW}$.year, which results from displacing OCGTs from the system (i.e. from storage replacing generation capacity while maintaining the prescribed supply adequacy standards).

In case of bulk energy storage there is additional small savings on interconnection and transmission network investment. In addition to achieving similar levels of savings in generation investment as bulk storage, distributed energy storage also generates savings in distribution network investment at the amount of around $£ 60 / \mathrm{kW}$.year. We again notice that the optimal volumes deployed are much higher than for bulk storage with similar cost.

Fig. 8 shows the results of the analysis carried out for years 2020 and 2050, which reveal (on the example of distributed storage of $10 \mathrm{GW}$ installed capacity) that the value of storage increases very significantly in the scenario with increasing contribution of renewable generation. The value increases predominantly through savings in operation cost and generation investment cost, which are also driven by progressively tighter $\mathrm{CO}_{2}$ emission constraints $(100$ and $50 \mathrm{~g} / \mathrm{kWh}$ in 2030 and 2050 , respectively).

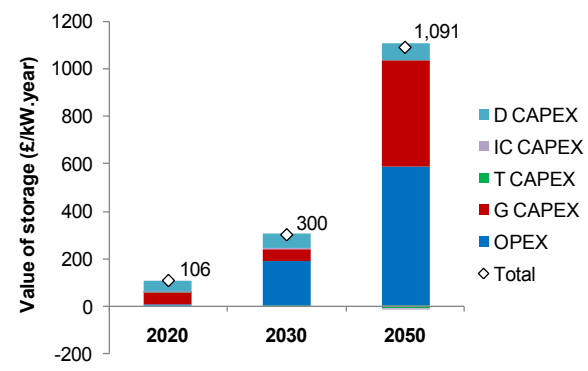

Fig. 8. Value of $10 \mathrm{GW}$ distributed storage for different time horizons

\section{Comparison between Bulk and Distributed Storage}

As discussed, storage technologies can facilitate more efficient operation and investment in network and generation reinforcement, bringing value across the entire electricity system. The proposed model can explicitly consider synergies and conflicts of storage resources being used for multiple purposes. For example, in the case of the UK system discussed in the paper, application of distributed storage in Scotland to reduce electricity peak load and the associated distribution network reinforcement cost, may lead to a higher capacity requirements of the transmission network between Scotland and England (given the dominant transmission power flows from Scotland to England). On the other hand, the application of distributed storage to reduce the cost of transmission may reduce savings associated with distribution network reinforcement cost. The proposed model will optimise the investment into energy storage technologies, their location and operation, trading-off between savings in operating cost and savings in system capacity cost. In most cases the additional value from distribution network investment savings are higher making distributed storage more valuable than bulk, as shown in Fig. 9, where a comparison is made with respect to values generated by bulk or distributed storage of fixed size $(2,5$ or $10 \mathrm{GW})$.

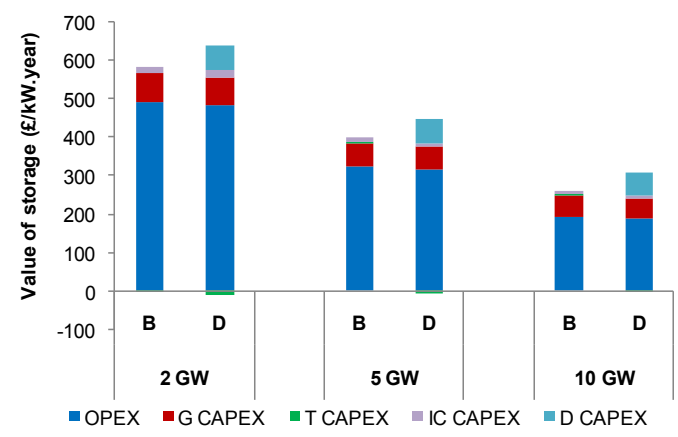

Fig. 9. Trade-off between cost savings for bulk (B) and distributed (D) storage

Most case studies presented in this paper assume either bulk or distributed storage was available for deployment in the system. Several additional studies have also been carried out to investigate the direct competition between bulk and distributed storage when both can be simultaneously added to the system. Fig. 10 shows the results of combined deployment of storage obtained for different cost levels of the two storage types - High ( $£ 200 / \mathrm{kW}$.year for bulk and $£ 250 / \mathrm{kW}$.year for distributed) and Low (£50/kW.year for bulk and $£ 100 / \mathrm{kW}$.year for distributed). ${ }^{2}$ The results of individual evaluations of bulk and distributed storage are also shown for reference. Circle sizes and the adjacent numbers indicate the level of deployment (in GW) of the two storage types.

It is evident that the optimal choice between bulk and distributed storage capacity is strongly driven by the cost assumptions. If both storage types are available at "Low" cost (although this is twice as high for distributed), the optimal deployment includes a relatively balanced mix of bulk and distributed storage (with the ratio of about 2:3), and the total storage capacity $(18.5 \mathrm{GW})$ that is greater than in either of the individual low-cost cases. If either of the storage types is available at low cost and the other one at high, the low-cost option strongly dominates the solution, and is chosen with a similar capacity as in the individual case. If on the other hand both bulk and distributed storage have high installation costs,

\footnotetext{
${ }^{2}$ Competition between low-cost distributed and high-cost bulk storage was not presented as studies suggested that the result would be virtually identical to the distributed-only case (distributed dominates bulk even when both are available at high cost).
} 
distributed is the preferred option, although at a much lower capacity than in the low-cost case. This is the consequence of the ability of distributed storage to capture benefits in avoided distribution investment cost, which is not possible using bulk storage.

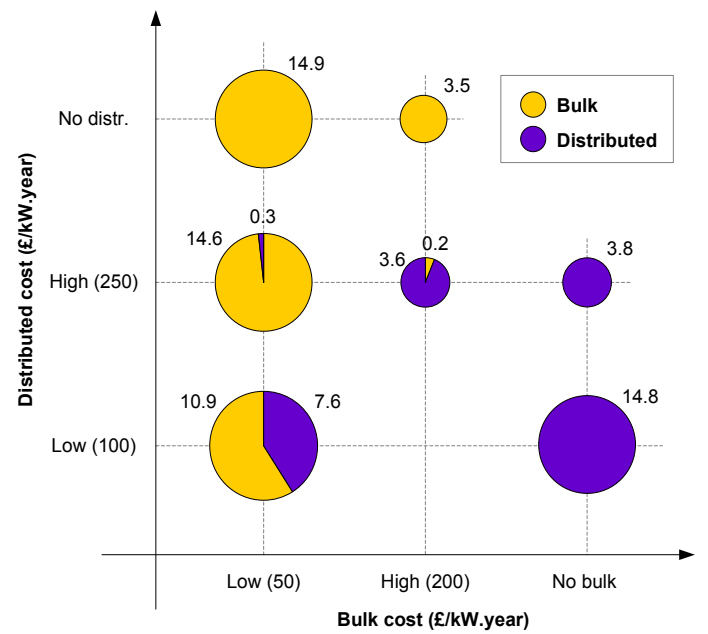

Fig. 10. Optimal volumes of bulk and distributed storage (in GW) when both are available for installation

\section{E. Impact of Storage Location}

The key differences in the role and value between bulk and distributed energy storage are further analysed in this section. Fig. 11 illustrates the trade-offs on an example spanning three consecutive days in Scotland for the year 2030. Scotland is presented here due to its high installed wind capacity. During the first day the wind output reaches the level of around $25 \mathrm{GW}$ and significantly exceeds demand; wind generation then drops rapidly to virtually zero during day 2 and remains very low during day 3 . The way energy storage located in Scotland will operate depends on its type (positive storage power in Fig. 11 refers to charging, while negative denotes discharging). Bulk energy storage is charged during high wind outputs, and discharged once the wind has dropped, except for a brief period with low demand during the second night. The resulting demand + storage profile is highly volatile with 22.5 GW peaks and even negative loads reaching $-5 \mathrm{GW}$, which are handled via the transmission link with England.

Distributed storage, on the other hand, follows a very different operating strategy. The charge and discharge pattern is less responsive to wind changes. Instead, distributed storage discharges in order to reduce peak load on all three days, even during periods of high wind. It also charges during periods of low wind if demand is low, resulting in a flattened demand profile at the level of about 9-10 GW. The surplus variable wind output exceeding $16 \mathrm{GW}$ in this case is exported to England, via a higher capacity transmission link, as shown in Fig. 11.
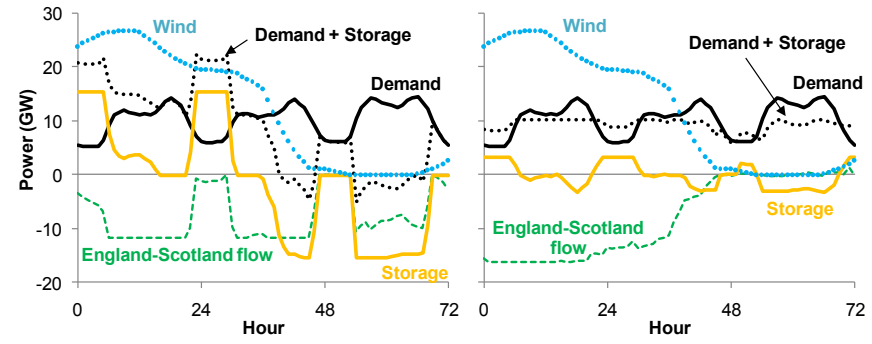

Fig. 11. Operating patterns of bulk (left) and distributed storage (right) in Scotland

Different operating patterns of bulk and distributed storage also have implications for their optimal location in the GB network. Fig. 12 shows the optimal allocation of storage capacity between Scotland and England by quantifying the part of storage capacity that is installed in Scotland, for the 6-hour storage duration.

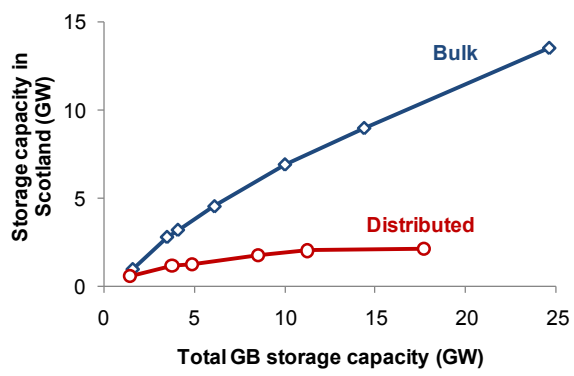

Fig. 12. Share of total storage capacity located in Scotland

Bulk storage is preferably placed in Scotland, where it supports the integration of wind and reduces costly reinforcement of additional transmission links with England. Only 20-30\% of the $10 \mathrm{GW}$ of bulk storage is deployed in the England region in this scenario. For distributed storage we observe an opposite trend; $80-85 \%$ of capacity is located in England and only $15-20 \%$ in Scotland. The dominant positioning of distributed storage in England is driven by a higher value accrued from avoided distribution network reinforcement.

\section{F. Competition with Other Flexible Technologies}

In addition to energy storage technologies, there are a range of other potential technology options available that could contribute to real-time system balancing, support the security of supply and mitigate investment in infrastructure reinforcement. In this context we quantified the value of storage in the presence of alternative technology solutions: (i) Interconnection: Increasing interconnection capacity between GB and Continental Europe and GB and Ireland may reduce balancing cost, by enhancing the ability of the system to accommodate renewables and enabling the sharing of longterm reserves; (ii) Flexible generation: Enhancing the flexibility of conventional generation (reducing minimum stable generation and increasing the frequency response capabilities of fossil fuel plant) would facilitate a more cost-effective integration of renewables; (iii) Demand-side response (DSR): Flexible demand side, in the form of electrified space and water heating in residential and commercial sectors, electrified transport sector and smart appliances in the household sector, may contribute to provision of energy arbitrage, reserve and frequency regulation services. 
The cost of interconnection capacity was assumed at the level of HVDC transmission shown in Table I. Although the model enables the cost of different flexible options to be specified, in the case studies presented in this section no cost was attributed to more flexible generation and DSR (due to lack of data), although the reduction in the value of storage driven by the presence of DSR and more flexible generation, is quantified

In general, these options will reduce the value of energy storage as indicated in Fig. 13, which presents the case of distributed storage with $24 \mathrm{~h}$ duration. We observe that the competing technologies reduce the absolute value of energy storage, mostly affecting its contribution to reducing operating costs and supporting real-time balancing.

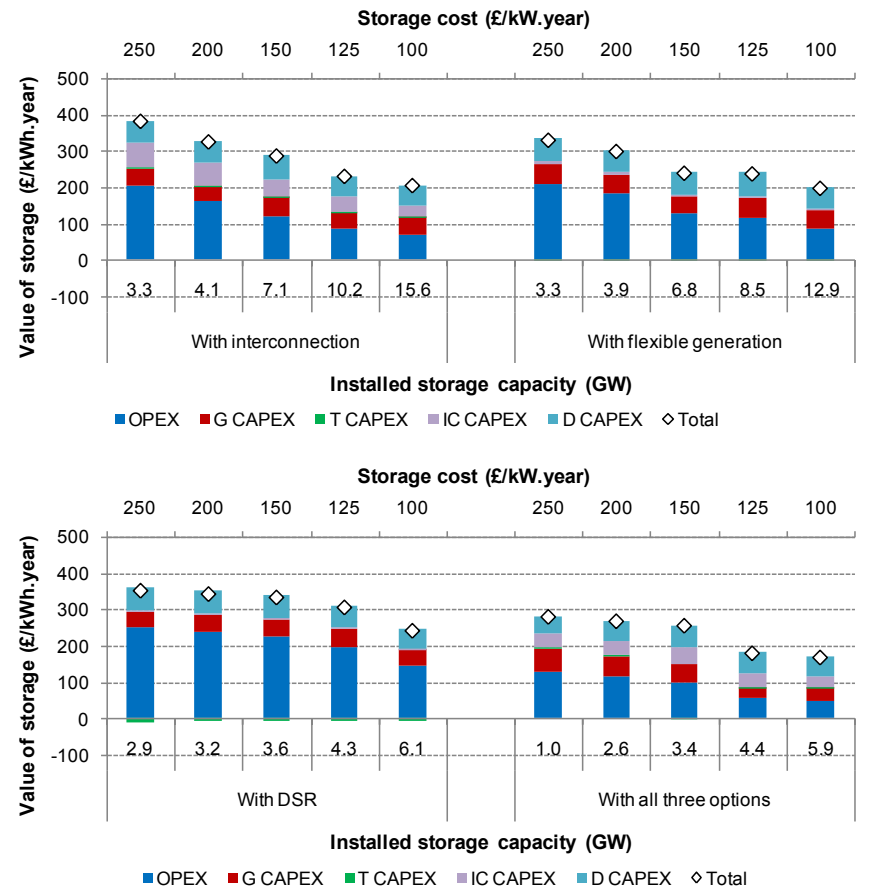

Fig. 13. Value of distributed storage in the presence of competing technologies

We also observe that the volumes of energy storage deployed in the presence of interconnection and more flexible generation are not significantly reduced compared to the baseline case presented in Fig. 7. In the case when interconnection expansion is allowed, there is even a slight increase in the optimal volume of storage in certain cases, suggesting synergistic effects rather than competition between storage and interconnection. On the other hand, if storage is competing with DSR, the deployed volumes of storage are several times lower than in the storage-only case. This is caused by the ability of responsive demand to flatten out the demand profile even before any distributed storage is added to the system, which reduces the opportunities for cost savings that storage could bring..

Finally, when distributed storage competes against all three flexible options, the deployed volumes further reduce compared to the DSR case, although not by much (with the exception of the highest cost level, where the volume drops by about 3 times). This supports the finding that the other two options, interconnections and flexible generation, compete less directly with energy storage.

In the above examples energy storage generates its largest share of value from savings in operational costs, which is driven by enhancing the ability of the system to absorb intermittent wind generation while dealing with wind uncertainty through deploying adequate levels of reserves. Given that wind uncertainty represents a critical driver for quantifying the system response and reserve requirements in (18) and (19), reducing the uncertainty of wind forecasting could reduce the value of storage for the system.

In order to illustrate the impact of improved wind forecasting, it was assumed that the 4-hr forecasting error (which is relevant for determining reserve requirements on the system given dynamic characteristics of generation) is reduced from $10 \%$ to $8 \%$ of installed wind capacity. In addition to the improved accuracy, it was also assumed that wind generation technology is able to contribute to frequency response when curtailed, which is in line with the expected improvements beyond 2020. The value of storage under these assumptions reduces significantly, as shown in Fig. 14.

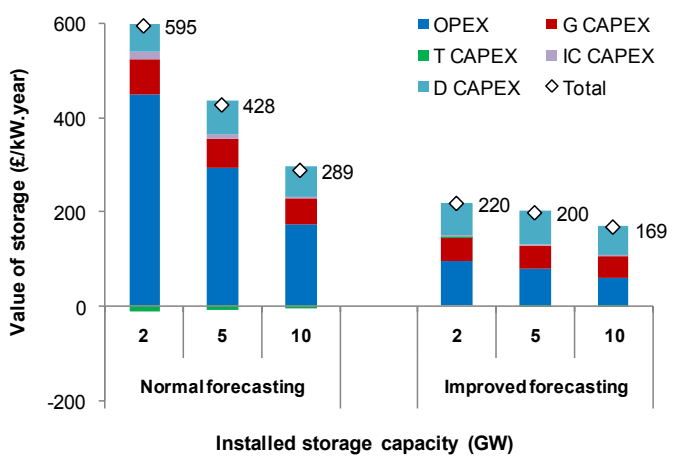

Fig. 14. Impact of improved wind forecasting on the value of distributed storage (6-hr duration)

\section{G. Impact of Storage Parameters}

\section{1) Storage efficiency}

All results presented so far assume a round-trip efficiency of storage of $75 \%$. Efficiency of different storage technologies available today can vary widely [31], and there is further uncertainty as to how the performance of different technologies may improve through research and development in the next few decades. A sensitivity analysis is therefore carried out for the following three efficiency levels: $50 \%, 75 \%$ and $90 \%$, and for the duration of $24 \mathrm{~h}$.

Fig. 15 illustrates that at low levels of deployment (i.e. high technology costs) the added value of increased efficiency is relatively low. However, this changes significantly if the costs of storage drop and deployment levels increase. We note that the optimal installed capacity of $90 \%$ efficient storage is twice as high as the optimal capacity of $50 \%$ efficient storage at the lowest cost point (corresponding to $£ 50 / \mathrm{kW}$.year for bulk and $£ 100 / \mathrm{kW}$.year for distributed storage). This follows from the fact that the marginal benefit of less efficient storage drops faster than the benefit of more efficient storage, particularly for high storage capacities. Faster decline of marginal benefits of less efficient storage results in a lower capacity at which the equilibrium is established between the marginal benefit of storage and its cost. 

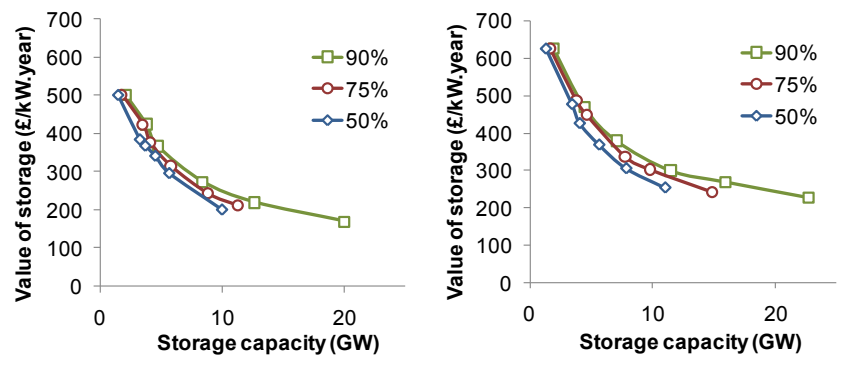

Fig. 15. Impact of efficiency on the capacity and value of bulk (left) and distributed storage (right)

\section{2) Value of additional storage duration}

No explicit cost has been assigned to the energy capacity in the case studies presented in this paper. However, a number of studies have been carried out for different storage durations, which allowed assessment of the value of additional energy by comparing the values for a given amount of storage capacity with different storage durations. The example of the $10 \mathrm{GW}$ storage shown in Fig. 16 demonstrates that the first additional hour of storage duration (i.e. an increase from $1 \mathrm{~h}$ to $2 \mathrm{~h}$ ) leads to the highest additional value, with further increases leading to diminishing returns.

Distributed storage initially gains significantly from an increase in storage duration (about $£ 34 / \mathrm{kWh}$.year). To make further increases in duration viable the cost has to significantly reduce, falling to less than $£ 1 / \mathrm{kWh}$.year for storage durations in excess of 6 hours. Value of additional duration for bulk storage is lower than distributed for storage durations below 6 hours.

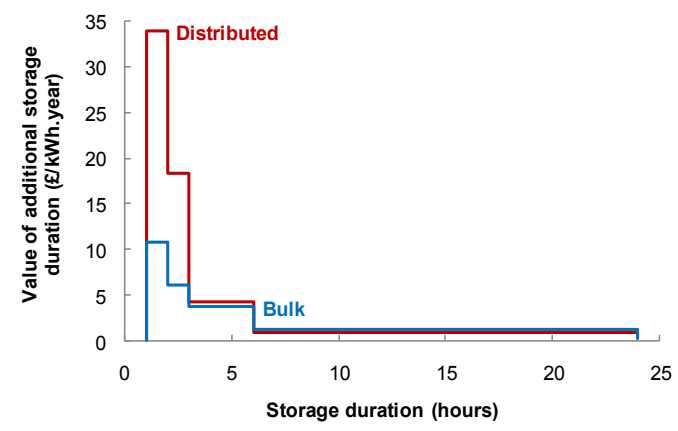

Fig. 16. Value of additional duration for $10 \mathrm{GW}$ storage

\section{CONCLUSIONS AND FUTURE WORK}

This paper presented a novel whole-systems approach to valuing the contribution of electricity storage for grid applications, revealing trade-offs between multiple services that energy storage is able to provide, which result in generally higher aggregate values for storage than those found in previous studies that considered such services in isolation. The case studies carried out demonstrate that energy storage can bring benefits across multiple sectors of electricity industry, including generation, transmission and distribution, while providing services to support real-time balancing of demand and supply, network congestion management and reducing the need for investment in system reinforcement. The proposed approach was applied to quantify the value of bulk and distributed energy storage and their market share in the future GB electricity system, including the analysis of competitiveness of energy storage against other technologies, such as flexible generation, interconnection and DSR. The model allows assessing the impact of various storage parameters on its value, including the importance of additional storage duration and storage efficiency.

Further work is needed to address the problem of "split benefits" associated with the development of appropriate market mechanisms to ensure that the investors in storage are adequately rewarded for delivering these diverse sources of value. Future improvements to the model presented in the paper may include the impact of operating patterns on storage cost and lifespan, as well as a more refined representation of the transmission network.

\section{REFERENCES}

[1] G. N. Bathurst, G. Strbac, "Trading wind generation in short term energy markets”, IEEE Trans. Power Syst., vol. 17, pp. 782-789, Aug. 2002.

[2] M. Black, G. Strbac, "Value of bulk energy storage for managing wind power fluctuations”, IEEE Trans. Energy Convers., vol. 22, pp. 197-205, Mar. 2007.

[3] J. Eyer and G. Corey, "Energy Storage for the Electricity Grid: Benefits and Market Potential Assessment", Sandia National Laboratories, Albuquerque, NM, Report SAND2010-0815, Feb. 2010. [Online]. Available: http://prod.sandia.gov/techlib/access-control.cgi/2010/100815.pdf.

[4] M. C. Such and C. Hill, "Battery energy storage and wind energy integrated into the Smart Grid", IEEE PES Innovative Smart Grid Technologies (ISGT), Washington, DC, Jan. 2012.

[5] C. A. Hill, M. C. Such, Dongmei Chen; J. Gonzalez, and W. M. Grady, "Battery Energy Storage for Enabling Integration of Distributed Solar Power Generation”, IEEE Trans. Smart Grid, vol. 3, pp. 850-857, Jun. 2012.

[6] A. Sturt, G. Strbac, "Efficient Stochastic Scheduling for Simulation of Wind-Integrated Power Systems", IEEE Trans. Power Syst., vol. 27, pp. 323-334, Feb. 2012.

[7] J. Garcia-Gonzalez, R. M. R. de la Muela, L. M. Santos, and A. M. Gonzalez, "Stochastic Joint Optimization of Wind Generation and Pumped-Storage Units in an Electricity Market", IEEE Trans. Power Syst., vol. 23, pp. 460-468, May 2008.

[8] D.J. Swider, "Compressed Air Energy Storage in an Electricity System With Significant Wind Power Generation", IEEE Trans. Energy Convers., vol. 22, pp. 95-102, Mar. 2007.

[9] S. O. Geurin, A. K. Barnes, and J. C. Balda, "Smart grid applications of selected energy storage technologies", IEEE PES Innovative Smart Grid Technologies (ISGT), Washington, DC, Jan. 2012.

[10] T. K. A. Brekken, A. Yokochi, A. von Jouanne, Z. Z. Yen, H. M. Hapke, and D. A. Halamay, "Optimal Energy Storage Sizing and Control for Wind Power Applications", IEEE Trans. Sustain. Energy, vol. 2, pp. 6977, Jan. 2011.

[11] P. Hu, R. Karki, and R. Billinton, "Reliability evaluation of generating systems containing wind power and energy storage", IET Generation Transmission \& Distribution, vol. 3, pp. 783-791, Aug. 2009.

[12] Peng Wang, Zhiyong Gao, and L. Bertling, "Operational Adequacy Studies of Power Systems With Wind Farms and Energy Storages", IEEE Trans. Power Syst., vol. 27, pp. 2377-2384, Nov. 2012.

[13] HyungSeon Oh, "Optimal Planning to Include Storage Devices in Power Systems”, IEEE Trans. Power Syst., vol. 26, pp. 1118-1128, Aug. 2011.

[14] H. Bludszuweit and J. A. Dominguez-Navarro, "A Probabilistic Method for Energy Storage Sizing Based on Wind Power Forecast Uncertainty", IEEE Trans. Power Syst., vol. 26, pp. 1651-1658, Aug. 2011.

[15] A. A. Thatte and Le Xie, "Towards a Unified Operational Value Index of Energy Storage in Smart Grid Environment", IEEE Trans. Smart Grid, vol. 3, pp. 1418-1426, Sep. 2012.

[16] A. Oudalov, D. Chartouni, and C. Ohler, "Optimizing a Battery Energy Storage System for Primary Frequency Control”, IEEE Trans. Power Syst., vol. 22, pp. 1259-1266, Aug. 2007.

[17] C. P. Nguyen and A. J. Flueck, "Agent Based Restoration With Distributed Energy Storage Support in Smart Grids", IEEE Trans. Smart Grid, vol. 3, pp. 1029-1038, Jun. 2012.

[18] N. S. Wade, P. C. Taylor, P. D. Lang, P. R. Jones, "Evaluating the benefits of an electrical energy storage system in a future smart grid", Energy Policy, vol. 38, pp. 7180-7188, Nov. 2010. 
[19] F. A. Chacra, P. Bastard, G. Fleury, and R. Clavreul, "Impact of energy storage costs on economical performance in a distribution substation", IEEE Trans. Power Syst., vol. 20, pp. 684-691, May 2005.

[20] Yixing Xu and C. Singh, "Adequacy and Economy Analysis of Distribution Systems Integrated With Electric Energy Storage and Renewable Energy Resources", IEEE Trans. Power Syst., Vol. 27, pp. 2332-2341, Nov. 2012.

[21] J. Mitra and M. R. Vallem, "Determination of Storage Required to Meet Reliability Guarantees on Island-Capable Microgrids With Intermittent Sources”, IEEE Trans. Power Syst., Vol. 27, pp. 2360-2367, Nov. 2012.

[22] M. Carrion and J. M. Arroyo, "A computationally efficient mixedinteger linear for-mulation for the thermal unit commitment problem", IEEE Trans. Power Syst., Vol. 21, pp. 1371-1378, Aug. 2006.

[23] A. Sturt, G. Strbac, "Value of stochastic reserve policies in low-carbon power systems", Proceedings of the Institution of Mechanical Engineers: Part O-Journal of Risk and Reliability, Vol. 226, pp. 51-64, Feb. 2012.

[24] C. K. Gan, M. Aunedi, V. Stanojevic, G. Strbac and D. Openshaw: "Investigation of the Impact of Electrifying Transport and Heat Sectors on the UK Distribution Networks", 21st International Conference on Electricity Distribution (CIRED), Frankfurt, Jun. 2011.

[25] D. Papadaskalopoulos, G. Strbac, P. Mancarella, M. Aunedi, V. Stanojevic, "Decentralized Participation of Flexible Demand in Electricity Markets - Part II: Application With Electric Vehicles and Heat Pump Systems", IEEE Trans. Power Syst., Vol. PP, May 2013.

[26] A. J. Wood and B. F. Wollenberg, Power generation, operation, and control, 2nd ed. New York; Chichester: Wiley, 1996.

[27] J. P. Green, S. A. Smith, G. Strbac, "Evaluation of electricity distribution system design strategies", IEE Proc. Gener. Transm. Distr., Vol: 146, pp. 53-60, Jan. 1999.

[28] C. K. Gan, P. Mancarella, D. Pudjianto, G. Strbac, "Statistical appraisal of economic design strategies of LV distribution networks", Electric Power Systems Research, Vol. 81, pp. 1363-1372, Jul. 2011.

[29] FICO Xpress optimization suite. [Online]. Available: http://optimization. fico.com.

[30] M. Castro, D. Pudjianto, P. Djapic, G. Strbac, "Reliability-driven transmission investment in systems with wind generation", IET Generation Transmission \& Distribution, Vol. 5, pp. 850-859, Aug. 2011.

[31] G. Strbac, M. Aunedi, D. Pudjianto, P. Djapic, F. Teng, A. Sturt, D. Jackravut, R. Sansom, V. Yufit, N. Brandon, "Strategic Assessment of the Role and Value of Energy Storage Systems in the UK Low Carbon Energy Future", report for Carbon Trust, Jun. 2012. [Online]. Available: http://www.carbontrust.com/resources/reports/technology/energystorage-systems-strategic-assessment-role-and-value.

[32] Electric Power Research Institute, "Electricity Energy Storage Technology Options: A White Paper Primer on Applications, Costs and Benefits", Dec. 2010. [Online]. Available:

http://www.epri.com/abstracts/pages/productabstract.aspx?ProductID $=0$ 00000000001020676 . 